\title{
A Novel Polychromatic Model for Light Dispersion
}

\author{
Samy Metari ${ }^{1}$ and François Deschênes ${ }^{2}$ \\ ${ }^{1}$ Université de Sherbrooke, Sherbrooke, Québec, Canada \\ Samy.Metari@usherbrooke.ca \\ ${ }^{2}$ Université du Québec à Rimouski, Rimouski, Québec, Canada \\ francois_deschenes@uqar.ca
}

\begin{abstract}
In computer vision, the majority of research works covering the subject of vision through participating media are based on the concept of single scattering of light rays. Only few works deal with multiple scattering and they do so under restrictive constraints. In this paper we introduce a new multiple-scattering based polychromatic model $(P M)$ for vision through participating media. This model involves two basic concepts, namely attenuation and ambient illumination. The resulting model can be applied to a wide range of media. For instance, it can be devoted to the modeling of atmospheric vision, underwater vision and vision through misty glass. We show that it can be used to accurately restore the original versions of degraded images taken through atmosphere. Experimental results confirm that the proposed model is both in good agreement with the theory, and useful in practice.
\end{abstract}

Keywords: Multiple scattering, participating media, vision model.

\section{Introduction}

In computer vision we are often dealing with images taken through participating media, more specifically through atmosphere, glass, and water. Those images reveal different visual effects caused, on the one hand, by the interaction of luminous rays reflected by scene points with particles suspended in the diffusing medium, and on the other hand, by the contribution of ambient illumination. For instance, we can quote the effects of glow around light sources of a scene imaged through bad weather condition, the defocused effect caused by vision through water, etc. One important challenge is the modeling of vision through participating media. During the last two decades, there was a growth of interests in this research field. Existing works can be classified in two great families, those based on single scattering of light 1377910 and those based on the concept of multiple scattering of light but under restrictive constraints [5]8]. In this paper, we introduce a new polychromatic model for the modeling of vision through participating media. The proposed model is based on the multiple scattering of light which is inspired by a kernel developed by Metari and Deschênes [5]. The proposed $P M$ can be used for a wide variety of applications namely, atmospheric

A. Elmoataz et al. (Eds.): ICISP 2010, LNCS 6134, pp. 471 478, 2010.

(C) Springer-Verlag Berlin Heidelberg 2010 
vision, underwater vision and vision through misty glass. For each type of application, the parameters of the generic $P M$ are mapped onto the physical factors of the considered medium. We are hence able to accurately simulate vision through different media and we accurately restore the original versions of degraded images taken through participating media. Experimental results confirm both the accuracy of our polychromatic model and its usefulness for computer vision.

In next section, we introduce the polychromatic model. In section 3 , we show how to derive applications driven models from the generic one. In section 4 , we present two applications based on the specific models. Finally, conclusion is given in section 5 .

\section{Vision through Participating Media - Polychromatic Model}

In imaging through participating media, the recorded intensity of a scene point can be seen as the sum of two different intensities originating from the attenuation of a light beam reflected by a scene point and the contribution of environmental illumination [6]. We start by describing the attenuation model.

\subsection{Attenuation Model}

The component $I_{a t t}$ of the intensity of a scene point due to the attenuation phenomenon through a participating medium is the fruit of the following process: First, the incident light beam $\Phi(\lambda)$ interacts with the reflectance properties $\rho(\lambda)$ of a scene point and gets reflected. The reflected light beam will be conveyed towards the imaging system. During this travel, it interacts with the medium particles. During this interaction, a physical phenomenon occurs, namely the multiple scattering of light beam by the particles suspended in the medium [2]. The multiple scattering phenomenon has as effect, the attenuation of the incident light beam. Once the scattered light beam reaches the imaging system, the recorded intensity is the one of the scene point due to attenuation phenomenon. If we refer to [5] and [8] the multiple scattering of a light beam through a participating medium can be modelled by the convolution product of this light beam with the considered Point Spread Function PSF. The above mentioned process is expressed by the following formula:

$$
I_{a t t}=\int_{\lambda} \Phi(\lambda) \rho(\lambda) f(\lambda) * P S F(\eta(\lambda), p) d \lambda,
$$

where $\lambda$ is the wavelength of incident light, $f(\lambda)$ is the chromatic filter which allows us to deal separately with colors, $p$ is a parameter function of the scene depth $z, \eta(\lambda)=\alpha(\lambda)+\beta(\lambda)$ is the attenuation parameter, where $\alpha(\lambda)$ is the absorption coefficient and $\beta(\lambda)$ is the scattering coefficient of the diffusion medium, and $\operatorname{PSF}(\eta(\lambda), p)$ is the multiple scattering model. Note that, the extinction factor $\eta(\lambda)$ is the gradual loss in intensity of any kind of flux through a medium. 
Hence, the radiance of an object point right in front of the observer $(p \simeq 0)$ is given by:

$$
I_{\text {att }}=\int_{\lambda} \Phi(\lambda) \rho(\lambda) f(\lambda) d \lambda=I_{\text {clear }},
$$

as $\lim _{p \rightarrow 0} \operatorname{PSF}(\eta(\lambda), p) \simeq \delta$ and the Dirac function $\delta$ is the neutral element of the convolution operator. $I_{\text {clear }}$ denotes the irradiance of scene point in the absence of medium attenuation.

\subsection{Ambient Illumination Model}

The multiple scattering of ambient illumination with particles suspended in the diffusing medium contributes to the apparent intensity of scene points. The contribution of ambient illumination $I_{a m b}$ is the result of the difference between its original value and the attenuated one along the line of sight $(L O S)[6]$. Note that the ambient illumination can have as origin a natural light source such as the sun or an artificial light source such as a lamp. The recorded intensity due to ambient illumination along the $L O S$ is given by:

$$
I_{a m b}=\int_{\lambda} \Psi(\lambda) f(\lambda) d \lambda-\int_{\lambda} \Psi(\lambda) f(\lambda) * \operatorname{PSF}(\eta(\lambda), p) d \lambda,
$$

with $\Psi(\lambda)$ denotes the radiance of ambient illumination. Hence, the contribution intensity of ambient illumination for an object right in front of the observer $(p \simeq 0)$ equals zero as $\lim _{p \rightarrow 0} \operatorname{PSF}(\eta(\lambda), p) \simeq \delta$.

In most situations the effects of both attenuation and ambient illumination phenomena are omnipresent. Thus the apparent intensity of a given scene point is the result of the contributions of both attenuation and ambient illumination:

$$
\begin{aligned}
I_{\text {scene }} & =\int_{\lambda} \Phi(\lambda) \rho(\lambda) f(\lambda) * \operatorname{PSF}(\eta(\lambda), p) d \lambda+\int_{\lambda} \Psi(\lambda) f(\lambda) d \lambda \\
& -\int_{\lambda} \Psi(\lambda) f(\lambda) * \operatorname{PSF}(\eta(\lambda), p) d \lambda .
\end{aligned}
$$

In practical situations, it is generally assumed that the considered medium is homogeneous. It results that the attenuation parameter $\eta(\lambda)$ is constant throughout the scene depth. Additionally, Narasimhan and Nayar [7] supposed that the attenuation factor is independent of the wavelength, i.e. $(\eta(\lambda)=\eta)$. Note that this assumption is valid in many cases such as vision through fog and dense haze [4]. Based on those assumptions, the analytical expression of our polychromatic model will given by:

$$
I_{\text {scene }}=\int_{\lambda} \Phi(\lambda) \rho(\lambda) f(\lambda) d \lambda * P S F(\eta, p)+\int_{\lambda} \Psi(\lambda) f(\lambda) d \lambda *(\delta-P S F(\eta, p)) .
$$

We chose to use as a basic PSF for the multiple scattering phenomenon, the kernel introduced by Metari and Deschênes [5]. Recall that many useful properties of the kernel such as Mellin transform, moments and Fourier transform 
are well-known. Additionally, the simplicity (absence of iterative process) of the analytical expression of the kernel makes possible the elaboration of real time applications. The analytical expression of the proposed PSF kernel is given by:

$$
P S F(x, y ; \eta, p)=\frac{e^{-\frac{\left(x^{2}+y^{2}\right)^{\frac{p}{2}}}{\left(\left.A(p, \eta)\right|^{p}\right.}}}{4 \Gamma^{2}\left(1+\frac{1}{p}\right) A(p, \eta)^{2}}, \quad A(p, \eta)=\left[\frac{\eta^{2} \Gamma\left(\frac{1}{p}\right)}{\Gamma\left(\frac{3}{p}\right)}\right]^{\frac{1}{2}} .
$$

In what follows we show how to derive a specific applications from the generic polychromatic model.

\section{Vision through Participating Media - Specific Applications}

In this section we introduce three specific applications of the polychromatic model. The first one is devoted to atmospheric vision, the second one to underwater vision and the last one to vision through misty glass.

\subsection{First Case: Atmospheric Vision}

In the case of atmospheric vision, the multiple scattering model is parameterized by two physical factors namely, the optical thickness $T$ and the forward scattering parameter $q$ [4]. The parameter $T$ is a dimensionless quantity equals to $\frac{3.912 z}{V}$ with $z$ the scene depth and $V$ the atmospheric visibility. The parameter $T$ is a simply scaled depth. The forward parameter $q$ indicates the type of weather condition.

The relationships between the parameters of the $P M$ and the atmospheric parameters are given by what follows:

$-\eta=\frac{1-q}{q}$.

$-p=k T$ with $k$ a given constant.

$-\Psi(\lambda)=L(\lambda)$ with $L(\lambda)$ the radiance of airlight.

\subsection{Second Case: Underwater Vision}

Three factors must be taken into account in modeling of underwater vision. The first one is the backscatter $B(\lambda)$ which represents the incident radiance that originates from the environmental illumination. The second one is the attenuation coefficient of the water $\gamma$ which is given by $\gamma=\gamma_{a b s}+\gamma_{s c a}$, where $\gamma_{a b s}$ is the absorption coefficient and $\gamma_{s c a}$ is the total scattering coefficient of the water. The last factor is the scene depth $z$. The mapping between the underwater parameters and the parameters of the $P M$ is given by:

$-\eta=\gamma$, i.e. $\alpha=\gamma_{a b s}$ and $\beta=\gamma_{s c a}$.

$-p=c z$, with $c$ a given constant number.

$-\Psi(\lambda)=B(\lambda)$. 


\subsection{Third Case: Vision through Misty Glass}

Additionally to underwater vision and atmospheric vision the polychromatic model is able to model vision through other participating media, such as tissu, glass, etc. In this section we propose a specific computer graphics application of the $P M$, namely the simulation of vision through misty glass. In this case, two important parameters must be considered, namely, the glass thickness $T_{g l a}$ and the extinction coefficient of the glass $\eta_{g l a}$. By analogy to the first two applications, we propose to use the following parameter mapping:

$-\eta=\eta_{g l a}$.

$-p=\kappa T_{\text {gla }}$, with $\kappa$ a given constant number.

\section{Applications}

In this section we present two sets of applications based on the proposed model. We begin by the simulation results of vision through participating media.

\subsection{Simulation of Atmospheric Vision}

In this experiment we simulate the effects of both rain (Fig 1.(b)) and fog (Fig 1.(c)) from a clear image of the scene (Fig 1.(a)). Note that the airlight magnitude is equal to $(R=179, G=182, B=181)$. Knowing that the atmospheric veil produced under foggy condition is more significant than the one produced under rainy condition [6], obtained results reveal a great realism. Thus, the density of atmospheric veil is proportional to the attenuation parameter $\eta$ of the medium.

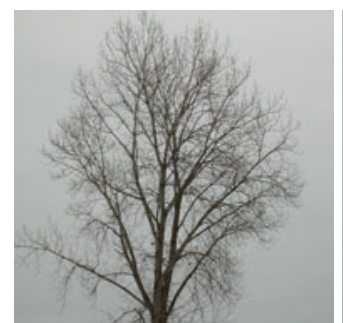

(a)

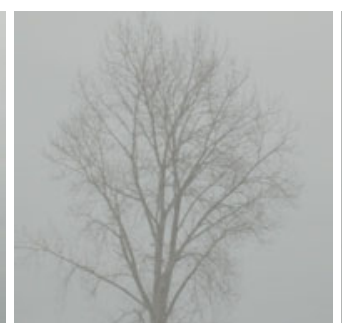

(b)

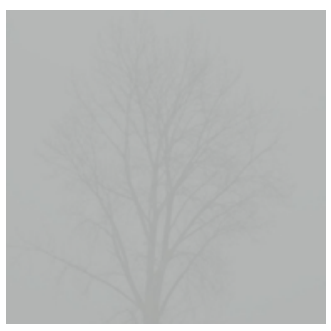

(c)

Fig. 1. a- original image, b- rainy image: $q=0.98$ and $k T=0.6$, c- foggy image: $q=0.9$ and $k T=0.6$. The size of images is $768 \times 756$.

\subsection{Simulation of Underwater Vision}

The following experiment is devoted to the simulation of underwater vision. More specifically, we simulate the effect of vision through water diluted with milk. 
Fig.2.(a) is the original image of the scene taken through clear water, using a digital camera (Canon 1-D professional), Fig 2. (b) is the original degraded image and Fig 2, (c) is the simulated one. The backscatter magnitude $(R=182, G=$ $211, B=183$ ) corresponds to the value of a pixel located at the background of the degraded image Fig 2(b). Note that the values attributed to both parameters $\gamma$ and $c z$ are the most appropriate in this case. In order to measure the quality of the obtained result, we use the Normalized Mean Squared Error (NMSE) as a measure between both images (b) and (c). Obtained result $(N M S E=0.0037)$ reveals that those images are almost identical.

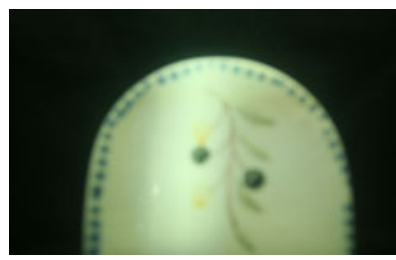

(a)

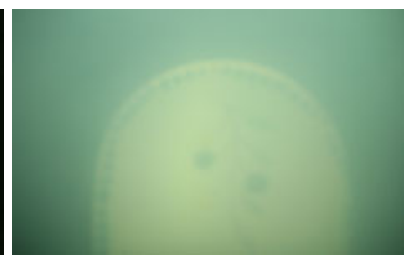

(b)



(c)

Fig. 2. Simulation of vision through diluted water. a- original clear image, b- original degraded image, c- simulated image: $\gamma=0.25$ and $c z=0.35$.

\subsection{Simulation of Vision through Misty Glass}

In the following set of experiments we simulate the effects of vision through different misty glasses. The magnitude of ambient illumination is equal to $(R=$ $220, G=220, B=220$ ). Note that the opacity of glasses increases from image (a) to image (c) due to the extinction factor $\eta_{g l a}$ values.

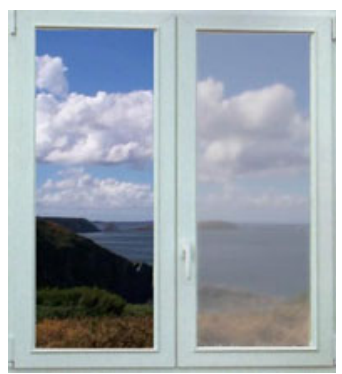

(a)

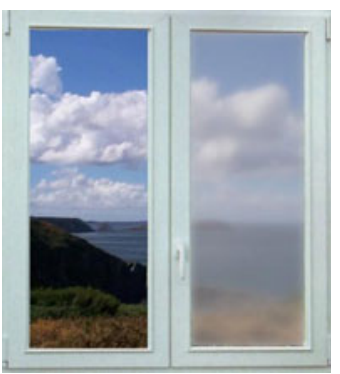

(b)

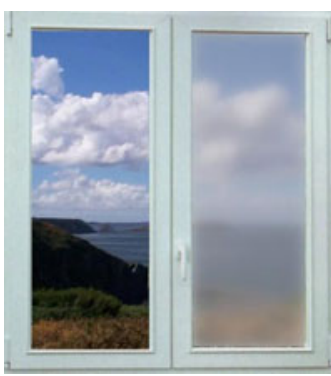

(c)

Fig. 3. Simulation of vision behind misty glasses. a- $\eta_{\text {gla }}=0.17$ and $\kappa T_{\text {gla }}=0.55$, b$\eta_{g l a}=0.53$ and $\kappa T_{g l a}=0.55, \mathrm{c}-\eta_{g l a}=1.22$ and $\kappa T_{g l a}=0.55$. The size of images is 407x450.

\subsection{Restoration of Weather Degraded Image}

This experiment is devoted to removing weather condition effects from a real degraded image. In this experiment, we consider a real hazy image (cf. Fig 4.(a)) 


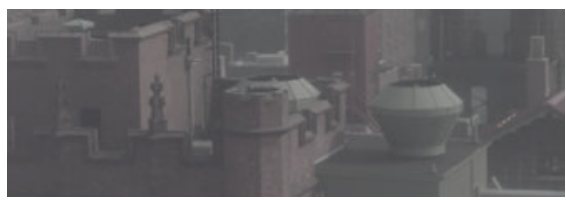

(a)

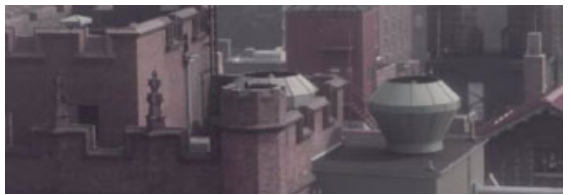

(b)

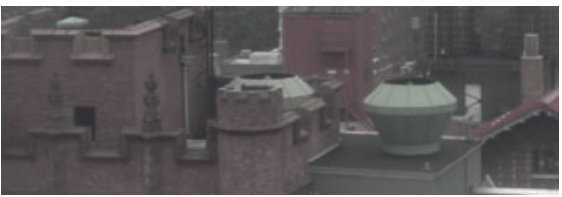

(c)

Fig. 4. Removing haze effect from degraded image: a- original hazy image, b- restored image, c- original clear image. The size of images is $656 \times 221$.

extracted from the Weather and Illumination Database (WILD) 1 . The associated metadata are the following: the atmospheric visibility $V=3$ miles and the near relative depth of the scene $z=1$ mile. Note that, we assume that $z$ is constant for the whole image pixels. Based on the given metadata we can easily identify both values of $q$ and $T$. Recall that $T=\frac{3.912 z}{V}$. The restoration process is done according to the following equation:

$$
I_{\text {clear }}=\mathcal{F}^{-1}\left[\frac{\mathcal{F}\left(I_{\text {scene }}\right)-\mathcal{F}\left(I_{\text {airlight }}\right)}{\mathcal{F}(\operatorname{PSF}(0.75,0.65))}\right],
$$

with $I_{\text {scene }}$ the acquired image, $I_{\text {clear }}$ the original one and $I_{\text {aitlight }}$ the airlight contribution. Note that $(\mathcal{F}(\cdot))$ denotes the Fourier transform and $\left(\mathcal{F}^{-1}(\cdot)\right)$ denotes the inverse Fourier transform.

According to [5], the analytical expression of the Fourier transform of the $P S F$ kernel is given by:

$$
\mathcal{F}(P S F(q, k T)) \simeq e^{-\frac{A\left(k T, \frac{1-q}{q}\right)^{k T}}{2^{k T}}\left(u^{2}+v^{2}\right)^{\frac{k T}{2}}} .
$$

The NMSE between Fig 4.(a) and Fig 4.(c) is equal to 0.12 while the NMSE between Fig 4.(b) and Fig 4.(c) equal 0.003. This confirms that the restored image is very close to the original one. Thus, the restoration process is done successfully.

\section{Conclusion}

Modeling vision through participating media (e.g. tissu, atmosphere, glass, water, etc) is closely related to modeling of multiple scattering of light rays by

${ }^{1}$ WILD is a data base of high quality images of outdoor scene elaborated by the Narasimhan and Nayar research group. 
particles suspended in these media. In this paper, we introduced a new polychromatic model inspired by the multiple scattering kernel introduced by Metari and Deschênes [5]. Based on the introduced model we derived three specific applications devoted to atmospheric vision, underwater vision and vision through misty glass. Based on those specific applications we elaborated two sets of experiments. The first one is devoted to the simulation of vision through different participating media. The second one is devoted to the restoration of real degraded images taken though the atmosphere. All of the experiments confirm both the accuracy of the proposed models and their usefulness in the research fields of computer vision and image processing.

\section{References}

1. Cozman, F., Krotkov, E.: Depth from scattering. In: International Conference on Computer Vision and Pattern Recognition, Puerto Rico, vol. 31, pp. 801-806 (1997)

2. Ishimaru, A.: Wave Propagation and Scattering in Random Media. IEEE Press, Los Alamitos (1997)

3. Lévesque, D., Deschênes, F.: Sparse scene structure recovery from atmospheric degradation. In: 17th International Conference on Pattern Recognition, vol. 1, pp. 84-87 (2004)

4. McCartney, E.J.: Optics of the Atmosphere. Scattering by molecules and particles. John Wiley and Sons, Chichester (1975)

5. Metari, S., Deschênes, F.: A new convolution kernel for atmospheric point spread function applied to computer vision. In: 11th International Conference on Computer Vision ICCV 2007, Rio de Janeiro, Brazil, pp. 1-8 (2007)

6. Middleton, W.E.K.: Vision through the Atmosphere. University of Toronto Press (1952)

7. Narasimhan, S.G., Nayar, S.K.: Vision and the atmosphere. International Journal of Computer Vision 48(3), 233-254 (2002)

8. Narasimhan, S.G., Nayar, S.K.: Shedding light on the weather. In: IEEE Computer Society Conference on Computer Vision and Pattern Recognition, USA, vol. 1, pp. 665-672 (2003)

9. Schechner, Y.Y., Karpel, N.: Clear underwater vision. In: IEEE Computer Society Conference on Computer Vision and Pattern Recognition, USA, vol. 1, pp. 536-543 (2004)

10. Tan, R.T.: Visibility in bad weather from a single image. In: IEEE Computer Society Conference on Computer Vision and Pattern Recognition, pp. 1-8 (2008) 